\title{
Perioperative management of immunosuppression in patients with rheumatoid arthritis
}

\author{
Michael D. George, MD MSCE ${ }^{1}$ and Joshua F. Baker, MD MSCE ${ }^{1,2}$ \\ 1. University of Pennsylvania, Division of Rheumatology \\ 2.Philadelphia Veterans Affairs Medical Center, Division of Rheumatology
}

\begin{abstract}
Purpose of review: Patients with rheumatoid arthritis are at increased risk of infection after surgery. Recent literature has provided more data and updated guidelines to guide the management of immunosuppression in the perioperative period.
\end{abstract}

Recent findings: Studies over the past few years have confirmed that patients with rheumatoid arthritis are at increased risk of infection after surgery. Patients treated with biologics are at greater risk of post-operative infection, but this risk might be explained by the comorbidities and greater disease severity often seen in these patients. Recent observational studies have suggested that interruption of biologic therapies before surgery may not be associated with better outcomes. Glucocorticoids, however, have consistently been found to be risk factors for infection. Recent guidelines from the American College of Rheumatology/American Association of Hip and Knee Surgeons recommend continuing conventional disease modifying drugs and holding biologics for one dosing interval before surgery.

Summary: Prolonged interruption of conventional and biologic therapies before surgery does not appear to substantially reduce infection risk. Guidelines now recommend continuing conventional DMARDs and holding biologics for just one dosing interval before surgery. Glucocorticoids are strongly associated with the risk of post-operative infection and should be minimized before surgery.

\section{Keywords}

perioperative management; biologics; DMARDs; glucocorticoids; rheumatoid arthritis

\section{Introduction}

Patients with rheumatoid arthritis (RA) frequently undergo surgery, particularly orthopedic surgery, even in the era of modern therapy [1,2]. The perioperative period is a high-risk time for infections such as surgical site infections and pneumonias. Arthroplasties and other surgeries that include prosthetic material are of are particular concern because of the

Corresponding Author: Michael D. George, University of Pennsylvania, Division of Rheumatology, 5 White Building, 3400 Spruce Street, Philadelphia, PA 19104, Phone: 215-662-2454, Michael.george@uphs.upenn.edu.

Conflicts of interest: Michael George has received a research grant from Bristol-Myers Squibb and consulting fees $<\$ 10,000$ from

Abbvie. Joshua Baker has received consulting fees $<\$ 10,000$ from Bristol-Myers Squibb. 
potential for infections of the prosthetic material. A frequent question facing clinicians is how to manage RA therapies in patients undergoing surgery. Until recently, there was little evidence and sparse, conflicting guidelines to inform management of immunosuppressive therapies in this setting. Studies published over the past several years, including updated guidelines, have expanded our understanding of the risk of infection in patients with RA undergoing surgery and the role of immunosuppressive medications, particularly biologic therapies and glucocorticoids. In this article we will summarize recent advances in the field and review recent guidelines. While uncertainty remains, these recent studies and guidelines allow a more rational and evidence-based approach to the patient with RA undergoing surgery.

\section{The risk of post-operative infection in patients with RA}

A number of studies have demonstrated that patients with RA are at increased risk of postoperative infection after orthopedic surgery [3-5]. Several recent studies have shown similar findings, suggesting this remains true in the modern era [6-9]. Cordtz et al. evaluated approximately 125,000 patients including 3913 patients with RA undergoing hip or knee arthroplasty in linked Danish registries and found a greater risk of prosthetic joint infection in patients with RA [HR 1.46 (1.13-1.88)] [6]. A similar study using inpatient data from the Nationwide Inpatient Sample found that patients with RA had significantly greater rates of infection, wound dehiscence, and systemic complications after hip and knee replacement compared to patients with OA [7]. Extending the data to other orthopedic surgeries, Horowitz et al. found that patients with RA had higher rates of post-operative infection after cervical fusion $(2.6 \%$ vs. $1.5 \%)$ [8]. It remains unclear, however, whether the increased risk of post-operative infection in RA is primarily related to medications, chronic systemic and local joint inflammation, or differences in comorbid conditions.

\section{The risk of post-operative infection in patients treated with biologic therapies}

Until recently, there was a lack of direct evidence to help guide the perioperative management of biologic therapies. These medications are known to be associated with infection risk in the non-surgical setting [10], and TNF inhibitors have been shown to have possible associations with skin and soft tissue infections and staph colonization [11,12]. The risk of infection seems to be particularly elevated in the first months after biologic initiation, although the higher risk might be related to greater disease activity or glucocorticoid dosing during this time or because the highest risk patients tend to experience infections early (depletion of susceptibles) [13].

Biologic therapies have different mechanisms of action, and determining whether infection risk differs between biologics remains an area of debate. Currently there is insufficient evidence to convincingly show a clinically meaningful difference in infection risk between biologics in the non-surgical setting, although this research continues to evolve [14,15]. Preliminary data suggests that the risk of infection after surgery is also similar in patients treated with different biologic therapies [16]. As TNF inhibitors remain the oldest and most 
commonly prescribed biologics, current data about perioperative risk with biologics comes largely from studies of these therapies.

Several older observational studies have compared the risk of post-operative infection in patients with RA treated with a TNF inhibitor vs. patients who are not receiving a biologic therapy. Goodman et al. conducted a meta-analysis that included 11 of these studies, including a total of 7991 patients with RA undergoing major orthopedic surgery [17]. Patients receiving a TNF inhibitor had higher rates of superficial and deep surgical site infection compared to patients not treated with a biologic [OR 2.47 (1.66 to 3.68)], although the included studies were small and the high potential for confounding could not be adequately addressed. A subsequent meta-analysis found similar results [18].

More recently, Cordtz et al. evaluated risk of prosthetic joint infection (PJI) in patients with RA treated with or without a biologic therapy within 90 days of hip or knee arthroplasty by linking several Danish registries [6]. In this study, 345 patients received a biologic within 90 days of surgery while 1601 patients with RA had no biologic treatment within 90 days of surgery. There were numerically more PJI in the biologic treated patients ( 2.8 vs. 1.9 per 100 person years in biologic vs. non-biologic treated patients), although differences were not statistically significant [adjusted HR $1.61(0.70-3.69)$ ]. It is important to note, however, that patients treated with a biologic DMARD had significantly greater disease activity and were more likely to be treated with glucocorticoids before surgery. Notably, rates of infection were greater in patients receiving glucocorticoids [(adjusted HR 2.12 (0.90-4.98)], and those with higher DAS28 disease activity [adjusted HR 2.00 (1.28-3.13)]. Glucocorticoids and disease activity (but not the use of biologics) were also associated with a greater risk of death within 1 year after surgery.

\section{Perioperative management of biologic therapies}

While prior studies have generated some concern for the risk of post-operative infection in patients treated with biologic therapies, the more important question facing clinicians is not whether patients receiving a biologic are at greater risk, but rather whether stopping biologic therapy before surgery will reduce that risk. Most studies to date have compared biologictreated and untreated patients, but these populations are quite different from each other in terms of disease activity, comorbidities, and other immunosuppression use (such as glucocorticoids). As a result, even the most sophisticated statistical methods are unlikely to be able to create completely comparable groups.

Because serious infections after surgery are relatively rare (approximately $1 \%$ risk of prosthetic joint infection after joint replacement), it is currently not feasible to conduct a trial randomizing patients to either stop or continue biologic therapy before surgery; detecting a modest difference in serious infections (e.g. 5 vs. $7 \%$ ) or of PJI (e.g. 1 vs. $2 \%$ ) would require a sample size of more than 4000 patients. Several recent observational studies, however, have begun to evaluate the timing of biologic therapies, providing some insight into the role of therapy interruptions before surgery (Table 1). 
Zahr et al. evaluated 6024 patients with RA treated with a DMARD, including 896 receiving a biologic, undergoing surgery using data from the Veteran's Administration [19]. Neither interruption of conventional DMARDs nor interruption of biologic DMARDs was associated with a reduced risk of post-operative infection. In this study, most DMARDs were not infusion therapies, and so timing was based on prescription data. Even though the authors conducted chart review to evaluate the performance of the timing algorithm, there remains a degree of uncertainty in the precise timing of treatment before surgery.

To address concerns about the accurate measurement of biologic timing prior to surgery, our group identified 4288 Medicare patients undergoing elective hip or knee arthroplasty who had received an infliximab infusion in the 6 months prior to surgery [20]. Infusions are coded as procedures in claims data and their timing can be precisely identified using the infusion administration date. We found that patients who received infliximab within 4 weeks of surgery had no difference in the risk of 30-day post-operative infection or 1-year prosthetic joint infection compared to patients in which infliximab had been last given at least one dosing interval (8-12 weeks) prior to surgery [OR 0.90 (0.60 to 1.34) and HR 0.98 $(0.52,1.87)$ respectively for the two outcomes]. The study controlled for a number of potential confounders, including comorbidities, healthcare utilization, and other medication use using propensity scores, although disease activity measures were not available.

An interesting study of 311 patients with inflammatory bowel disease undergoing abdominal surgery evaluated associations between infliximab levels and post-operative infection risk [21]. High infliximab levels $\geq 3 \mu \mathrm{g} / \mathrm{mL}$ were associated with greater infection risk, but only in patients with Crohn's disease and not among those with ulcerative colitis. The timing and dose of infliximab treatment were not available and ability to account for confounders including disease severity was limited. We are not aware of similar studies evaluating drug levels and post-operative risk patients with RA.

There is limited data to guide the timing of non-TNF biologic therapies. Small studies of patients treated with tocilizumab [22] and rituximab [23] undergoing surgery have too few outcomes to adequately assess risk factors for infection. Results from a French abatacept registry evaluated 263 patients receiving intravenous abatacept undergoing surgery and found that the timing of abatacept was not associated with the risk of complications, although the sample size was small and the power to detect differences was limited [24]. Preliminary results from our study of 1537 Medicare patients treated with intravenous abatacept undergoing total hip or knee arthroplasty showed similar results; patients receiving abatacept within 4 weeks of surgery had similar rates of post-operative infection and readmission compared to patients in which abatacept was last given more than 4 weeks (1 dosing interval) prior to surgery [25].

The results of these studies provide some evidence that prolonged biologic treatment interruption may not substantially improve outcomes after surgery, at least for infliximab. The existing studies are observational and even relatively large observational studies cannot definitely prove that continuing treatment is safe. The data overall, however, suggests that the benefit of interrupting therapy, if any, is likely to be fairly small. 


\section{Perioperative risk associated with glucocorticoids}

While substantial attention has been paid to biologic therapies in the perioperative period, relatively little attention has been paid to glucocorticoids. When they are evaluated, however, glucocorticoids are frequently found to be associated with a substantial increase in the risk of infection after surgery [6,20,26-28]. The risk of infection is dose dependent, although it is unclear whether there is a "safe" dose. In our infliximab study and in preliminary results of a larger study of biologic-treated patients, we found that the risk of post-operative infection was approximately doubled in patients receiving more than 10mg per day of prednisone in the three months prior to surgery $[16,20]$. Thus, while the benefit of stopping biologic drugs in the perioperative setting has been debated, there seems to be consensus that the risk of infection with higher doses of glucocorticoids is substantial. These results suggest that prolonged interruption of biologics may in fact be counter-productive if patients experience disease flares that require glucocorticoid treatment. These data also support a goal of reducing glucocorticoid use prior to surgical interventions whenever possible.

\section{Recommendations from recent guidelines}

A major advance has been the creation of guidelines from the American College of Rheumatology (ACR) in collaboration with the American Association of Hip and Knee Surgeons (AAHKS) for the perioperative management of medications in patients with rheumatic disease undergoing elective hip or knee arthroplasty [29]. These guidelines recommend continuing conventional DMARDs methotrexate, leflunomide, sulfasalazine, and hydroxychloroquine throughout the perioperative period without interruption. Influential in these guidelines was evidence that infection risk with methotrexate is low in patients not undergoing surgery [30], and data from small randomized trials showing similar or increased risk of post-operative infections with discontinuation of conventional DMARDs before surgery [31,32].

These guidelines also address the timing of biologic therapy before surgery. Because of the known risk of infection associated with biologic therapies from non-surgical settings, the panel recommended holding biologics for one dosing interval prior to surgery. The dosing interval was used rather than half-life because the half-life was not felt to correlate as well with the duration of action. According to these guidelines, a patient receiving infliximab every 8 weeks would last receive the medication 9 weeks before surgery, while a patient receiving adalimumab every two weeks would receive the medication 3 weeks (15-21 days) before surgery. The guidelines recommend restarting biologics 14 days after surgery if the surgical site is healing well and there are no signs of local or systemic infection. Updated guidelines on biologic DMARD safety from the British Society of Rheumatology have taken a similar approach, recommending interruption for one dosing interval for most biologics (except for 3 weeks for subcutaneous tocilizumab), although the option is also given to hold for 5 half-lives for higher risk surgeries [33].

An interesting and novel aspect of ACR/AAHKS guidelines was the inclusion of a patient panel, 11 patients with inflammatory arthritis who had undergone arthroplasty [34]. This 
patient panel placed much higher importance on avoiding perioperative infections, a quite reasonable perspective given the potential devastating effects of prosthetic joint infections.

\section{Weighing the risks and benefits of stopping biologic therapy in the patient with RA}

The guidelines have provided a starting point from which to consider minimizing risk in the perioperative period, yet it is important to interpret the evidence in the context of each individual patient. It may seem that the conservative approach would be to stop biologics before surgery, even though current evidence has not shown a clear benefit with treatment interruption. This approach is reasonable for many patients, but for some patients stopping biologics may not be the best approach to minimize risk. For example, patients who frequently experience disease flares requiring treatment with higher doses of glucocorticoids may have better outcomes if biologic therapies are continued, rather than interrupted, during the perioperative period. For certain patients, concerns about disease flares, which are common in the post-operative period [35], may outweigh infection concerns, especially for low-risk surgeries without prosthetic material. In contrast, patients in long-term disease remission but with multiple infectious complications in the past may be more likely to benefit from treatment interruption.

Fortunately, holding treatment for one dosing interval before surgery and restarting 14 days after surgery, as suggested by ACR guidelines, leads to only a brief treatment interruption for most patients. The more recent observational data certainly supports the hypothesis that longer interruptions are likely not necessary.

It deserves highlighting that higher doses of glucocorticoids appear to contribute substantially more to post-operative infection risk than biologic therapies. While ACR guidelines recommend avoiding elective surgery in patients requiring $>20 \mathrm{mg}$ per day of prednisone [29], risk appears to be increased even at lower doses. Every effort should be made to taper glucocorticoids to the lowest possible dose before surgery. Patients on higher doses should consider deferring elective surgeries until their disease can be more effectively managed. A suggested approach to the patient with RA undergoing surgery, based on existing data and guidelines, is shown in Figure 1.

\section{Conclusion}

Clinical studies over the past few years have improved our understanding of the appropriate perioperative management of biologic therapies, although much uncertainty remains. Recent data has confirmed that patients with RA are at greater risk of infection after surgery, although medications likely only explain a part of that risk. Guidelines now support continuation of conventional DMARDs throughout the perioperative period and recommend brief interruptions of biologic therapies for one dosing interval before surgery. More recent observational data provides supportive data that prolonged discontinuation of biologics does not improve outcomes and that limiting glucocorticoid exposure before surgery is of major importance. Taken together, these data can help guide the management of patients with RA undergoing surgery as the field continues to evolve. 


\section{Acknowledgements}

Financial support and sponsorship: Michael George is supported by the Rheumatology Research Foundation Scientist Development Award and the National Institute of Arthritis and Musculoskeletal and Skin Diseases 1K23AR073931-01. Joshua Baker has been supported by a VA Clinical Science Research \& Development Career Development Award (IK2 CX000955) and Merit Award (I01 CX001703). The contents of this work do not represent the views of the Department of the Veterans Affairs or the United States Government.

Funding: Michael George is supported by the Rheumatology Research Foundation Scientist Development Award and the National Institute of Arthritis and Musculoskeletal and Skin Diseases K23-AR073931-01. Joshua Baker has been supported by a VA Clinical Science Research \& Development Career Development Award (IK2 CX000955) and Merit Award (I01 CX001703). The contents of this work do not represent the views of the Department of the Veterans Affairs or the United States Government.

\section{References}

1. Shourt CA, Crowson CS, Gabriel SE, et al. Orthopedic Surgery Among Patients with Rheumatoid Arthritis 1980-2007: A Population-based Study Focused on Surgery Rates, Sex, and Mortality. The Journal of Rheumatology 2012;39:481-5. doi:10.3899/jrheum.111056 [PubMed: 22247350]

2. Cordtz RL, Hawley S, Prieto-Alhambra D, et al. Incidence of hip and knee replacement in patients with rheumatoid arthritis following the introduction of biological DMARDs: an interrupted timeseries analysis using nationwide Danish healthcare registers. Ann Rheum Dis 2018;77:684-9. doi: 10.1136/annrheumdis-2017-212424 [PubMed: 29247125]

3. Stundner O, Chiu Y-L, Sun X, et al. Perioperative outcomes in patients with rheumatoid versus osteoarthritis for total hip arthroplasty: a population-based study. Clin Exp Rheumatol 2013;31:88995. [PubMed: 24237847]

4. Stundner O, Danninger T, Chiu Y-L, et al. Rheumatoid Arthritis vs Osteoarthritis in Patients Receiving Total Knee Arthroplasty: Perioperative Outcomes. The Journal of Arthroplasty 2014;29:308-13. doi:10.1016/j.arth.2013.05.008 [PubMed: 23764034]

5. Ravi B, Croxford R, Hollands S, et al. Increased Risk of Complications Following Total Joint Arthroplasty in Patients With Rheumatoid Arthritis: Increased Risk of Specific Complications in TJA Recipients With RA. Arthritis \& Rheumatology 2014;66:254-63. doi:10.1002/art.38231 [PubMed: 24504797]

** 6. Cordtz RL, Zobbe K, Højgaard P, et al. Predictors of revision, prosthetic joint infection and mortality following total hip or total knee arthroplasty in patients with rheumatoid arthritis: a nationwide cohort study using Danish healthcare registers. Ann Rheum Dis 2018;77:281-8. doi: 10.1136/annrheumdis-2017-212339 This Danish study showed greater risk of PJI in patients with RA vs. OA. Among RA patients, biologic use was associated with a non-significant increase in infection risk. Glucocorticoids and disease activity were more strongly associated with infection risk. [PubMed: 29097373]

7. Kurdi AJ, Voss BA, Tzeng TH, et al. Rheumatoid Arthritis vs Osteoarthritis: Comparison of Demographics and Trends of Joint Replacement Data from the Nationwide Inpatient Sample. Am J Orthop 2018;47. doi:10.12788/ajo.2018.0050

8. Horowitz JA, Puvanesarajah V, Jain A, et al. Rheumatoid Arthritis Is Associated With an Increased Risk of Postoperative Infection and Revision Surgery in Elderly Patients Undergoing Anterior Cervical Fusion. Spine 2018;43:E1040-4. doi:10.1097/BRS.0000000000002614 [PubMed: 29481378]

9. Salt E, Wiggins AT, Rayens MK, et al. Moderating effects of immunosuppressive medications and risk factors for post-operative joint infection following total joint arthroplasty in patients with rheumatoid arthritis or osteoarthritis. Semin Arthritis Rheum 2017;46:423-9. doi:10.1016/ j.semarthrit.2016.08.011 [PubMed: 27692433]

10. Singh JA, Cameron C, Noorbaloochi S, et al. Risk of serious infection in biological treatment of patients with rheumatoid arthritis: a systematic review and meta-analysis. Lancet 2015;386:25865. doi:10.1016/S0140-6736(14)61704-9 [PubMed: 25975452]

11. Galloway JB, Mercer LK, Moseley A, et al. Risk of skin and soft tissue infections (including shingles) in patients exposed to anti-tumour necrosis factor therapy: results from the British 
Society for Rheumatology Biologics Register. Ann Rheum Dis 2013;72:229-34. doi:10.1136/ annrheumdis-2011-201108 [PubMed: 22532633]

12. Varley CD, Deodhar AA, Ehst BD, et al. Persistence of Staphylococcus aureus colonization among individuals with immune-mediated inflammatory diseases treated with TNF- $a$ inhibitor therapy. Rheumatology (Oxford) 2014;53:332-7. doi:10.1093/rheumatology/ket351 [PubMed: 24173434]

13. Strangfeld A, Eveslage M, Schneider M, et al. Treatment benefit or survival of the fittest: what drives the time-dependent decrease in serious infection rates under TNF inhibition and what does this imply for the individual patient? Ann Rheum Dis 2011;70:1914-20. doi:10.1136/ard. 2011.151043 [PubMed: 21791449]

14. Yun H, Xie F, Delzell E, et al. Comparative Risk of Hospitalized Infection Associated With Biologic Agents in Rheumatoid Arthritis Patients Enrolled in Medicare. Arthritis \& Rheumatology (Hoboken, NJ) 2016;68:56-66. doi:10.1002/art.39399

15. Rutherford AI, Subesinghe S, Hyrich KL, et al. Serious infection across biologic-treated patients with rheumatoid arthritis: results from the British Society for Rheumatolgy Biologics Register for Rheumatoid Arthritis. Ann Rheum Dis Published Online First: 283 2018. doi:10.1136/ annrheumdis-2017-212825

* 16. George MD, Baker JF, Winthrop K, et al. OP0228 Comparative risk of biologic therapies and risk of glucocorticoids in patients with rheumatoid arthritis undergoing elective arthroplasty. Presented at the European League Against Rheumatism annual meeting, June 2018. Annals of the Rheumatic Diseases 2018;77:163. This abstract presents preliminary data showing similar risk of post-operative infection in patients with RA treated with biologics. Glucocorticoids, especially prednisone $>10 \mathrm{mg} /$ day, were associated with greater risk of infection

17. Goodman SM, Menon I, Christos PJ, et al. Management of perioperative tumour necrosis factor a inhibitors in rheumatoid arthritis patients undergoing arthroplasty: a systematic review and metaanalysis. Rheumatology (Oxford) 2016;55:573-82. doi:10.1093/rheumatology/kev364\[PubMed: 26447162]

18. Mabille C, Degboe Y, Constantin A, et al. Infectious risk associated to orthopaedic surgery for rheumatoid arthritis patients treated by anti-TNFalpha. Joint Bone Spine 2017;84:441-5. doi: 10.1016/j.jbspin.2016.06.011 [PubMed: 27663754]

* 19. Abou Zahr Z, Spiegelman A, Cantu M, et al. Perioperative use of anti-rheumatic agents does not increase early postoperative infection risks: a Veteran Affairs' administrative database study. Rheumatol Int 2015;35:265-72. doi:10.1007/s00296-014-3121-0 [PubMed: 25187198] This Veteran's Administration study showed no improvement in the risk of post-operative infection in patients with RA who had interrupted biologic therapy or who had interrupted a conventional DMARD, although the definition of interruption was based on prescription data for most patients.

** 20. George MD, Baker JF, Hsu JY, et al. Perioperative Timing of Infliximab and the Risk of Serious Infection After Elective Hip and Knee Arthroplasty. Arthritis Care Res (Hoboken) 2017;69:1845-54. doi:10.1002/acr.23209 [PubMed: 28129484] In this Medicare study of 4288 infliximab-treated patients undergoing hip or knee replacement, infection risk was similar in patients who received infliximab within 4 weeks of surgery compared to patients receiving infliximab 4-8 weeks or 8-12 weeks before surgery. Glucocorticoid dose > 10mg/day was associated with greater infection risk.

* 21. Lau C, Dubinsky M, Melmed G, et al. The impact of preoperative serum anti-TNFa therapy levels on early postoperative outcomes in inflammatory bowel disease surgery. Ann Surg 2015;261:487-96. doi:10.1097/SLA.0000000000000757 [PubMed: 24950263] Patients with Crohn's disease, but not ulcerative colitis, who had infliximab levels $\geq 3 \mu \mathrm{g} / \mathrm{mL}$ had higher rates of infection after abdominal surgery. Infliximab dose and timing was not available, however, and relevance to patients with RA is unclear.

22. Momohara S, Hashimoto J, Tsuboi H, et al. Analysis of perioperative clinical features and complications after orthopaedic surgery in rheumatoid arthritis patients treated with tocilizumab in a real-world setting: results from the multicentre TOcilizumab in Perioperative Period (TOPP) study. Mod Rheumatol 2013;23:440-9. doi:10.1007/s10165-012-0683-0 [PubMed: 22684398]

23. Godot S, Gottenberg J-E, Paternotte S, et al. Safety of surgery after rituximab therapy in 133 patients with rheumatoid arthritis: data from the autoimmunity and rituximab registry. Arthritis Care Res (Hoboken) 2013;65:1874-9. doi:10.1002/acr.22056 [PubMed: 23754822] 
* 24. Latourte A, Gottenberg J-E, Luxembourger C, et al. Safety of surgery in patients with rheumatoid arthritis treated by abatacept: data from the French Orencia in Rheumatoid Arthritis Registry. Rheumatology (Oxford) 2017;56:629-37. doi:10.1093/rheumatology/kew476 [PubMed: 28053274] In this French registry study, the timing of abatacept before surgery was not associated with the risk of post-operative complications, although the analysis was limited by small sample size

* 25. George M, Baker J, Winthrop K, et al. OP0227 Timing of abatacept before elective arthroplasty and post-operative outcomes. Presented at the European League Against Rheumatism annual meeting, June 2018. Annals of the Rheumatic Diseases 2018;77:162-3. doi:10.1136/ annrheumdis-2018-eular.3549 [PubMed: 28928270] This abstract presents preliminary results from Medicare data showing no difference in the risk of infection among patients receiving intravenous abatacept within 4 weeks of hip and knee arthroplasty compared to patients receiving abatacept $4-8$ weeks or $\geq 8$ weeks before surgery.

26. Somayaji R, Barnabe C, Martin L. Risk factors for infection following total joint arthroplasty in rheumatoid arthritis. Open Rheumatol J 2013;7:119-24. doi:10.2174/1874312920131210005 [PubMed: 24454587]

27. Michaud K, Fehringer EV, Garvin K, et al. Rheumatoid arthritis patients are not at increased risk for 30-day cardiovascular events, infections, or mortality after total joint arthroplasty. Arthritis Res Ther 2013;15:R195. doi:10.1186/ar4385 [PubMed: 24252350]

28. Gilson M, Gossec L, Mariette X, et al. Risk factors for total joint arthroplasty infection in patients receiving tumor necrosis factor a-blockers: a case-control study. Arthritis Research \& Therapy 2010;12:R145. doi:10.1186/ar3087 [PubMed: 20637100]

** 29. Goodman SM, Springer B, Guyatt G, et al. 2017 American College of Rheumatology/American Association of Hip and Knee Surgeons Guideline for the Perioperative Management of Antirheumatic Medication in Patients With Rheumatic Diseases Undergoing Elective Total Hip or Total Knee Arthroplasty. Arthritis \& Rheumatology (Hoboken, NJ) 2017;69:1538-51. doi: 10.1002/art.40149Updated guidelines recommend holding biologic therapies for one dosing interval prior to hip and knee replacement and restarting 14 days after surgery in the absence of signs of infection. Conventional disease modifying drugs can be continued without interruption.

30. Lopez-Olivo MA, Siddhanamatha HR, Shea B, et al. Methotrexate for treating rheumatoid arthritis. Cochrane Database Syst Rev 2014;:CD000957. doi:10.1002/14651858.CD000957.pub2 [PubMed: 24916606]

31. Grennan DM, Gray J, Loudon J, et al. Methotrexate and early postoperative complications in patients with rheumatoid arthritis undergoing elective orthopaedic surgery. Ann Rheum Dis 2001;60:214-7. [PubMed: 11171680]

32. Tanaka N, Sakahashi H, Sato E, et al. Examination of the risk of continuous leflunomide treatment on the incidence of infectious complications after joint arthroplasty in patients with rheumatoid arthritis. J Clin Rheumatol 2003;9:115-8. doi:10.1097/01.RHU.0000062514.54375.bd [PubMed: 17041441]

* 33. Holroyd CR, Seth R, Bukhari M, et al. The British Society for Rheumatology biologic DMARD safety guidelines in inflammatory arthritis. Rheumatology (Oxford) Published Online First: 189 2018. doi:10.1093/rheumatology/key298Updated guidelines from the British Society of Rheumatology on the use of biologics before surgery are similar to updated guidelines from the American College of Rheumatology.

* 34. Goodman SM, Miller AS, Turgunbaev M, et al. Clinical Practice Guidelines: Incorporating Input From a Patient Panel. Arthritis Care Res (Hoboken) 2017;69:1125-30. doi:10.1002/acr.23275 [PubMed: 28620968] This paper describes inclusion of a patient panel in guideline development, with patients placing much greater weight on the importance of preventing post-operative infections rather than preventing disease flares after joint replacement surgery.

* 35. Goodman SM, Bykerk VP, DiCarlo E, et al. Flares in Patients with Rheumatoid Arthritis after Total Hip and Total Knee Arthroplasty: Rates, Characteristics, and Risk Factors. J Rheumatol 2018;45:604-11. doi:10.3899/jrheum.170366 [PubMed: 29545451] 


\section{Key Points:}

- $\quad$ Emerging data suggests that stopping biologic therapy before surgery may not be associated with a substantially lower risk of post-operative infection

- Glucocorticoids are a major risk factor for post-operative infection

- Recent guidelines from the American College of Rheumatology recommend continuing conventional DMARDs throughout the perioperative period and stopping biologic DMARDs for one dosing interval before major elective surgery 


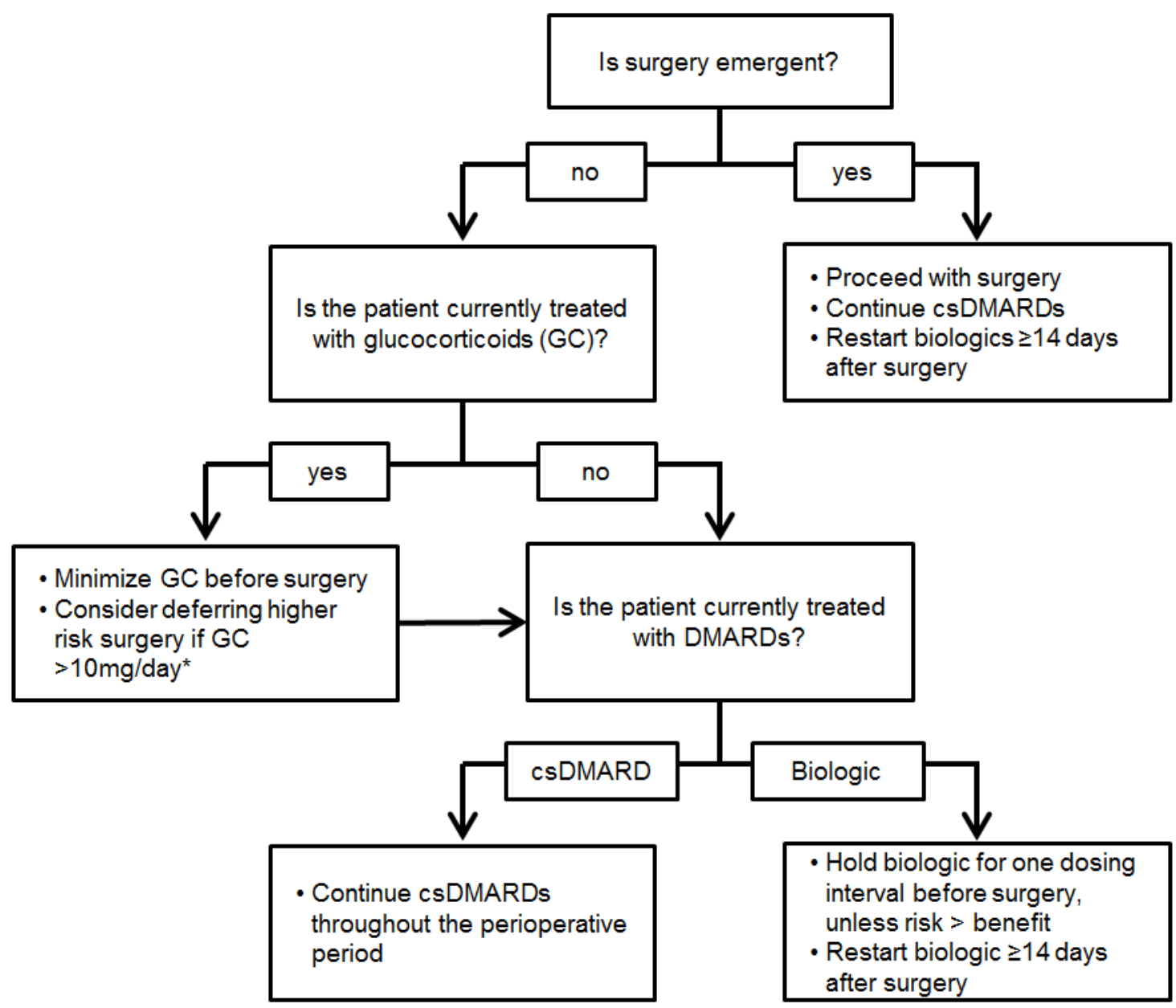

Figure 1: Suggested approach to patients with rheumatoid arthritis undergoing major surgery Suggestions based on recommendations for csDMARDs and biologics from 2017 American College of Rheumatology/American Association of Hip and Knee Surgeons perioperative guidelines [29] and observational data on glucocorticoid risk [6,20,26-28].

* Surgeries with prosthetic material and abdominal surgeries are of particular concern csDMARD = conventional synthetic disease modifying anti-rheumatic drug (methotrexate, leflunomide, hydroxychloroquine, sulfasalazine); $\mathrm{GC}=$ glucocorticoids 
Table 1:

Recent observational studies evaluating biologic timing before surgery

\begin{tabular}{|c|c|c|c|c|}
\hline Study & Population & Treatment & Results & Limitations \\
\hline Zahr, Rheum Int 2015[19] & $\begin{array}{l}896 \text { RA; various } \\
\text { surgeries }\end{array}$ & Various biologics & $\begin{array}{c}\text { Stop vs. continue: Infection OR } \\
1.52(0.88-2.62)\end{array}$ & $\begin{array}{c}\text { Potential } \\
\text { misclassification of } \\
\text { biologic timing, no } \\
\text { inclusion of disease } \\
\text { activity }\end{array}$ \\
\hline George, AC\&R 2017[20] & $\begin{array}{l}\text { 4288 RA, PsA, } \\
\text { AS, IBD; hip/ } \\
\text { knee arthroplasty }\end{array}$ & Infliximab & $\begin{array}{c}\text { Stop vs. continue (8-12 weeks } \\
\text { vs. < 4 weeks): Infection OR } \\
1.11(0.75-1.67)^{*} \text {; PJI HR } 1.02 \\
(0.54-1.92)^{*}\end{array}$ & $\begin{array}{c}\text { No inclusion of disease } \\
\text { activity }\end{array}$ \\
\hline Latourte, Rheumatology 2017[24] & $\begin{array}{l}263 \mathrm{RA} \text {; various } \\
\text { surgeries }\end{array}$ & Abatacept & $\begin{array}{l}\text { Median time before surgery in } \\
\text { patients with and without } \\
\text { complications: } 5.1 \text { vs. } 5.6 \text { weeks } \\
\text { (p }=0.41)\end{array}$ & Limited power \\
\hline George, ARD 2018 (abstract)[25] & $\begin{array}{l}1537 \mathrm{RA} ; \text { hip/ } \\
\text { knee arthroplasty }\end{array}$ & Abatacept & $\begin{array}{l}\text { Stop vs. continue ( } \geq 8 \text { weeks vs. } \\
<4 \text { weeks): Infection OR } 1.11 \\
(0.69-1.79) ; \text { PJI HR } 0.71 \\
(0.25-2.03)\end{array}$ & $\begin{array}{c}\text { No inclusion of disease } \\
\text { activity }\end{array}$ \\
\hline $\begin{array}{l}\text { Momohara, Mod Rheumatol } \\
\text { 2013[22] }\end{array}$ & $\begin{array}{l}169 \mathrm{RA} ; \\
\text { orthopedic } \\
\text { surgery }\end{array}$ & Tocilizumab & $\begin{array}{c}3 \text { surgical site infections, unable } \\
\text { to evaluate associations between } \\
\text { timing and infection }\end{array}$ & $\begin{array}{l}\text { Too few outcomes to } \\
\text { assess timing }\end{array}$ \\
\hline Godot, AC\&R 2013[23] & $\begin{array}{l}140 \mathrm{RA} \text {; various } \\
\text { surgeries }\end{array}$ & Rituximab & $\begin{array}{l}\text { Median time between rituximab } \\
\text { infusion and surgery in patients } \\
\text { with and without complications: } \\
6.43 \text { vs. } 6.49 \text { months }(p=1.0)\end{array}$ & Limited power \\
\hline Lau, Ann Surg 2015[21] & $\begin{array}{l}331 \mathrm{IBD} \\
\text { abdominal } \\
\text { surgery }\end{array}$ & Infliximab & $\begin{array}{l}\text { Infliximab level } \geq 3 \text { vs. }<3 \\
\mu \mathrm{g} / \mathrm{mL}: \text { Infection OR } 2.86 \\
(1.01-8.08), \text { only in Crohn's } \\
\text { disease }\end{array}$ & $\begin{array}{l}\text { No infliximab timing or } \\
\text { dose, no disease } \\
\text { activity, different } \\
\text { results in Crohn's and } \\
\text { ulcerative colitis }\end{array}$ \\
\hline
\end{tabular}

* OR and HR from this study inverted to provide results for stopping vs. continuing. OR = odds ratio, HR = hazard ratio, RA = rheumatoid arthritis, PsA = psoriatic arthritis, $\mathrm{AS}=$ ankylosing spondylitis, $\mathrm{IBD}=$ inflammatory bowel disease, $\mathrm{PJI}=$ prosthetic joint infection 